\title{
IMPACTO DA ETIOLOGIA E SITUAÇÕES ESPECIAIS NO TEMPO DE ESPERA EM LISTA E MELD DE ALOCAÇÃO NO TRANSPLANTE DE FÍGADO NO ESTADO DO PARANÁ
}

\section{Impact of the etiology and special situations on waiting time on list and MELD of allocation in liver transplantation in the state of Paraná}

\author{
Fábio Silveira, Fábio Porto Silveira, Cassia Regina Sbrissia Silveira, Ricardo Teles Schulz, \\ Ana Sofia Jaramillo Montero, Henrique Cesar Higa
}

\begin{abstract}
RESUMO
O sistema MELD está sedimentado no Brasil. O aumento do MELD para alocação, a influência de diferentes etiologias e o desequilíbrio na lista, secundários à concessão de situações especiais têm suscitado discussões para modernização do sistema. No Brasil, cada estado é responsável pela organização da captação de órgãos, resultando em um sistema heterogêneo. Objetivo: Análise do tempo de espera e MELD necessário para transplante, o impacto das diferentes etiologias e concessão de situações especiais no âmbito do estado do Paraná. Método: Análise de 1248 transplantes de fígado adulto entre 2010 e 2018, estratificados conforme a etiologia, MELD e tempo de espera. Resultados: Idade de $52 \pm 11,4$ anos. Tempo de espera em lista de $112 \pm 197$ dias. $83,78 \%$ dos casos transplantaram em período inferior a seis meses. Situações especiais foram concedidas em $8,25 \%(n=103)$ dos casos; não houve diferença significativa no tempo de espera em lista no grupo com $(110 \pm 107)$ e sem $(112 \pm 197)$ situação especial. MELD do transplante $(19,69 \pm 7,86)$, MELD corrigido $(22,24 \pm 6,42)$. Apesar de diferente entre grupos de etiologia, o MELD não apresentou padrão de crescimento com o passar do tempo. A etiologia álcool $(27,08 \%)$ e infecções virais $(23,56 \%)$ foram as mais frequentes. $29,75 \%$ dos transplantes foram realizados com MELD acima de $75 \%$; não houve modificação das etiologias mais comuns nesse subgrupo. A oferta de órgãos cresceu de 8,9 pmp (2010) para 47,7 pmp (2017). Conclusão: $O$ impacto do progressivo aumento do MELD e tempo de espera em lista, secundários à concessão de situações especiais não são observados na lista de espera no estado do Paraná. Essa discrepância provavelmente é secundária ao contínuo crescimento, de maneira quase paralela ao número de doadores e transplantes realizados.
\end{abstract}

Descritores: Transplante de Fígado; Listas de Espera; Tecnologia Aplicada a Listas de Espera; Indicador de Risco.

Instituição:

Centro Digestivo e Transplante de Órgãos / Hospital do Rocio. Campo Largo/PR, Brasil.

\section{Correspondência:}

Fábio Silveira

Estrada da Cachoeira 1000, casa 9 - Campo Largo/PR (41) 99691-1866

fabiosilveira@cdto.med.br

\section{INTRODUÇÃO}

A adoção da política de alocação de órgãos por critério de gravidade da doença, através do uso do escore de modelo de doença hepática terminal (MELD), foi adotada em 2006 no Brasil, após experiência inicial nos Estados Unidos. ${ }^{1}$ Diferentes etiologias podem refletir diferentes escores de MELD, sendo que, em algumas delas, o escore subestima seu risco de mortalidade.1,2 Para ajustar esse risco de mortalidade em lista de espera, ${ }^{3}$ situações ditas especiais recebem pontos de ajuste ao seu escore MELD. 
Após a adoção do modelo MELD, diversos sistemas de transplantes observaram progressivo aumento do índice de gravidade necessário para a obtenção do órgão, ${ }^{4}$ alguns relatando aumento de mortalidade pósoperatória, ${ }^{5}$ outros não. ${ }^{1} \mathrm{O}$ aumento da frequência de concessão de situações especiais (carcinoma hepatocelular, prurido intratável, ascite refratária, síndrome hepato-pulmonar, entre outros) tem sido relatado como um ponto de desequilíbrio do sistema, sendo que esse grupo de pacientes tem apresentado menor tempo de espera em lista, com progressiva "inflação" do MELD. ${ }^{6}$

O Sistema Nacional de Transplantes estratifica a lista de espera em um caráter estadual, sendo que cada estado é responsável pela organização das Organizações de Procura de Órgãos (OPOs). Esse caráter organizacional é nitidamente heterogêneo, com diferentes taxas de doação nos mais diferentes estados brasileiros. O estado do Paraná é, atualmente, o líder nacional em doadores (47,7 pmp), com progressivo crescimento nos últimos anos, muito acima da média nacional de 17pmp. ${ }^{7}$

O objetivo do presente estudo foi a análise do tempo de espera em lista e do MELD necessário para transplante, conforme as diferentes etiologias, no âmbito do Sistema Estadual de Transplantes do Paraná.

\section{MÉTODO}

O objeto de estudo foram todos os transplantes de fígado de doador falecido realizados no estado do Paraná, no período de 2010 a 2018. Os dados foram levantados da base de dados da Secretaria Estadual de Transplantes do Paraná e do Sistema Nacional de Transplantes. Critério de exclusão foi idade menor que 18 anos. Os dados coletados incluíram data do transplante, idade e sexo do receptor, MELD no momento da entrada na lista de espera (MELD), MELD laboratorial na alocação do órgão (MELDTX), MELD corrigido pela situação especial na alocação do órgão (MELDTx corrigido), tempo de espera em dias, situações especiais, urgência e etiologia da doença. Para facilitar análise, agrupamos as etiologias autoimune, cirrose biliar primária, secundária e colangite esclerosante primária sob a alcunha de doenças colestáticas. Variáveis qualitativas foram avaliadas pelo teste exato de Fisher ou qui-quadrado. Para variáveis quantitativas, análise de variância (ANOVA) de dados paramétricos e teste de Kruskalwallis para não paramétricos. A coleta e análises de dados foram realizadas pelo software Epidemiológico Epilnfo' $^{\mathrm{TM}}$ (versão 7.2.2.16, Center for Disease Control and Prevention). ${ }^{8}$ Teste estatísticos com significância quando $p<0,05$.

\section{RESULTADOS}

Um total de 1271 transplantes foram analisados, sendo $1248(98,19 \%)$ realizados em receptores adultos. Média de idade foi de $52,48 \pm 11,48$, com $69,79 \%(n=871)$ do sexo masculino. Tempo médio de espera em lista foi de $112 \pm 197$ dias. Escore MELD, MELDTx e MELDTx corrigido estão demonstrados na tabela 1.

Tabela 1: Características da população estudada

\begin{tabular}{lcccc}
\hline & Média & Q25 & Mediana & Q75 \\
\hline Idade & $52,48 \pm 11,48$ & 46 & 54 & 61 \\
$\begin{array}{l}\text { Tempo espera } \\
\text { (dias) }\end{array}$ & $112 \pm 197$ & 12 & 49 & 134 \\
$\begin{array}{l}\text { MELD } \\
\text { MELDTX }\end{array}$ & $18,42 \pm 7,57$ & 13 & 18 & 22 \\
$\begin{array}{l}\text { MELDTX } \\
\text { corrigido }\end{array}$ & $22,24 \pm 6,42$ & 18 & 21 & 24 \\
\hline
\end{tabular}

Tempo de espera em lista menor do que seis meses foi observado em 83,78\% $(n=1043)$ dos casos.

Observaram-se diferenças significativas no tempo de espera, MELDTx e MELDTx corrigido entre as diferentes etiologias (Tabela 2). A etiologia mais frequente foi 0 álcool, seguida das infecções virais pelo vírus B e C. A relação da etiologia com o MELDTx está demonstrada na Figura 1.

Os valores médios do MELDTx e MELDTx corrigidos foram estatisticamente diferentes na sequência dos anos estudados (tabela 3 ), porém sem um padrão evidente de crescimento (figura 2). A frequência de pacientes transplantados situados acima do percentil 75 (Q75) do MELDTx corrigido atingiu o pico em 2014 (45,83\%), com decréscimo nos anos subsequentes. (Figura 2).

Em média, 29,75\% dos casos foram transplantados com MELD médio acima do Q75. As doenças que levaram ao transplante no subgrupo do Q75 foram a cirrose alcoólica em $24,67 \%(n=75)$, cirrose decorrente da infecção pelo vírus da hepatite $B$ ou C em 16,45\% $(n=50)$ e cirrose criptogênica em $13,82 \%(n=42)$.

Houve aumento progressivo da oferta de doadores efetivos no período estudado, com diferenças no tempo de espera em lista conforme o ano considerado. (tabela 4) 
Situação especial e de urgência foram concedidas em $8,17 \%(n=102)$ e $3,76 \%(n=47)$ dos casos, respectivamente. O motivo mais comum de concessão de situação especial foi hepatocarcinoma em $81,55 \%(n=84)$ dos casos.
A situação de urgência mais comumente solicitada foi por insuficiência hepática fulminante em $48,98 \%$ $(n=24)$ dos casos, seguida de retransplante por não funcionamento primário em $28,57 \%(n=14)$ dos casos.

Tabela 2: Características da população estudada segundo etiologia da doença

\begin{tabular}{lccccc}
\hline & Frequência & $\mathbf{n}$ & Tempo espera & MELD Tx & $\begin{array}{c}\text { MELD Tx } \\
\text { corrigido }\end{array}$ \\
\hline Cirrose alcoólica & $27,08 \%$ & 338 & $87,3 \pm 142,1$ & $20,4 \pm 6,16$ & $20,9 \pm 5,7$ \\
Cirrose decorrente vírus B ou C & $23,56 \%$ & 294 & $146,9 \pm 271,0$ & $18,28 \pm 6,9$ & $20,7 \pm 5,6$ \\
Cirrose criptogênica & $11,54 \%$ & 144 & $106,7 \pm 153,2$ & $22,27 \pm 7,88$ & $22,52 \pm 5,85$ \\
Câncer primário de fígado & $10,66 \%$ & 133 & $107,7 \pm 81,5$ & $11,48 \pm 4,76$ & $22,45 \pm 3,3$ \\
Cirrose por DHGNA & $6,41 \%$ & 80 & $87,43 \pm 166,2$ & $22,27 \pm 7,88$ & $23,32 \pm 6,9$ \\
Hepatite fulminante & $2,24 \%$ & 28 & $3,71 \pm 5,2$ & $36,28 \pm 9,95$ & $37,28 \pm 8,76$ \\
Doenças colestáticas & $10,26 \%$ & 128 & $130,87 \pm 5,2$ & $21,59 \pm 6,06$ & $22,4 \pm 5,41$ \\
Outros & $8,25 \%$ & 103 & $126,35 \pm 266,9$ & $19,63 \pm 10,29$ & $25 \pm 7,9$ \\
\end{tabular}

Tabela 3: Índices de gravidade estratificados por ano de transplante

\begin{tabular}{cccc}
\hline Ano transplante & MELD Tx & MELD Tx corrigido & Q75 \\
\hline 2010 & $20,63 \pm 11,05$ & $21,18 \pm 11,84$ & $40 \%(n=2)$ \\
2011 & $17,66 \pm 6,66$ & $19,52 \pm 5,83$ & $10 \%(n=4)$ \\
2012 & $18,84 \pm 7,28$ & $21,38 \pm 6,55$ & $21,79 \%(n=17)$ \\
2013 & $18,24 \pm 7,91$ & $21,44 \pm 6,20$ & $25 \%(n=19)$ \\
2014 & $19,96 \pm 9,14$ & $24,39 \pm 7,14$ & $45,83 \%(n=33)$ \\
2015 & $17,82 \pm 7,03$ & $22,33 \pm 5,58$ & $32,97 \%(n=30)$ \\
2016 & $19,67 \pm 7,62$ & $22,31 \pm 5,88$ & $30,06 \%(n=52)$ \\
2017 & $22,07 \pm 8,06$ & $23,22 \pm 6,95$ & $33,77 \%(n=78)$ \\
2018 & $19,41 \pm 7,55$ & $21,70 \pm 6,03$ & $27,71 \%(n=69)$ \\
& $P<0,05$ & $P<0,05$ & \\
\hline
\end{tabular}


Figura 1: MELD tx médio segundo a etiologia.

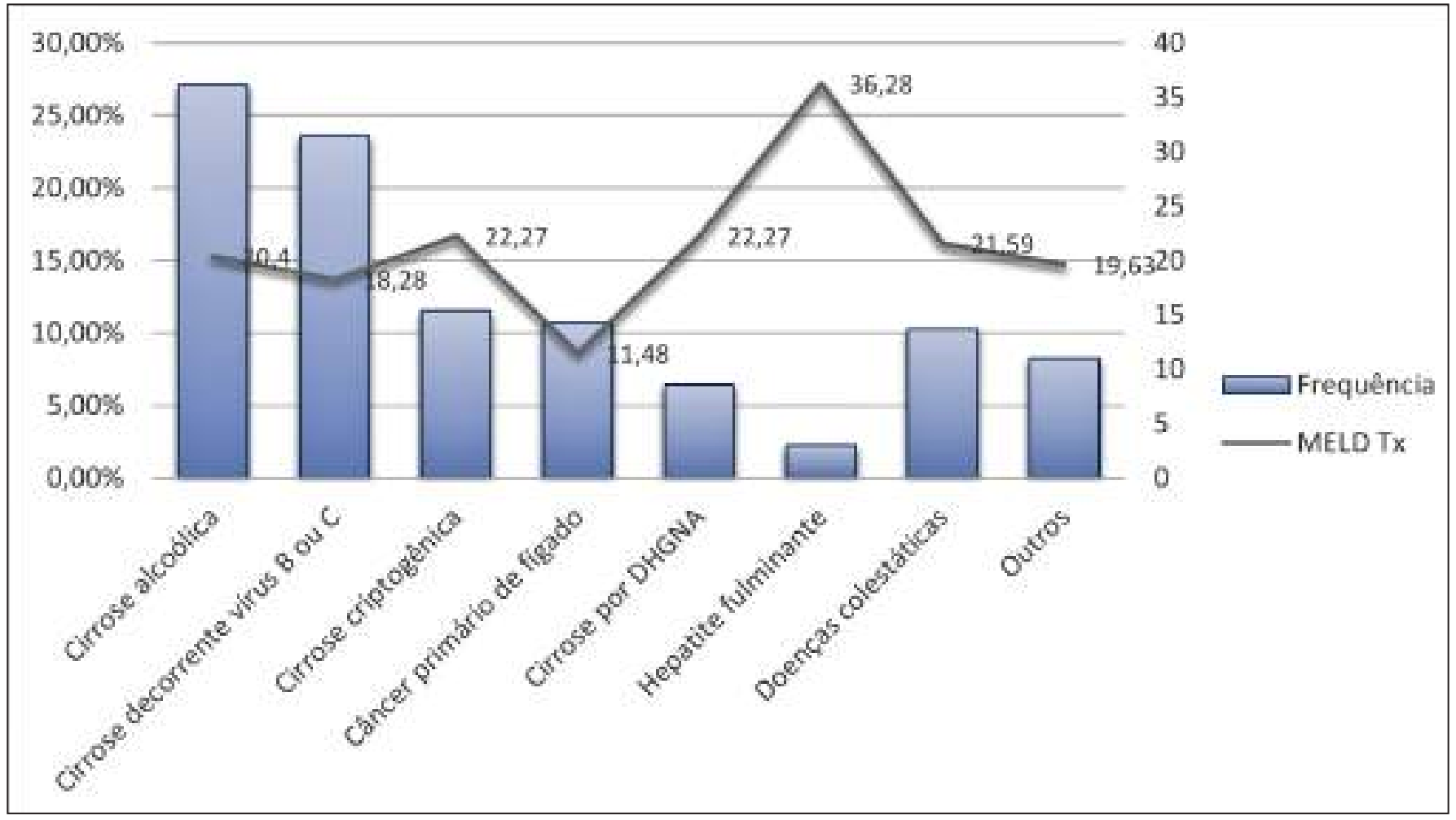

Figura 2: Demonstração gráfica da evolução do índice de gravidade por ano de transplante.

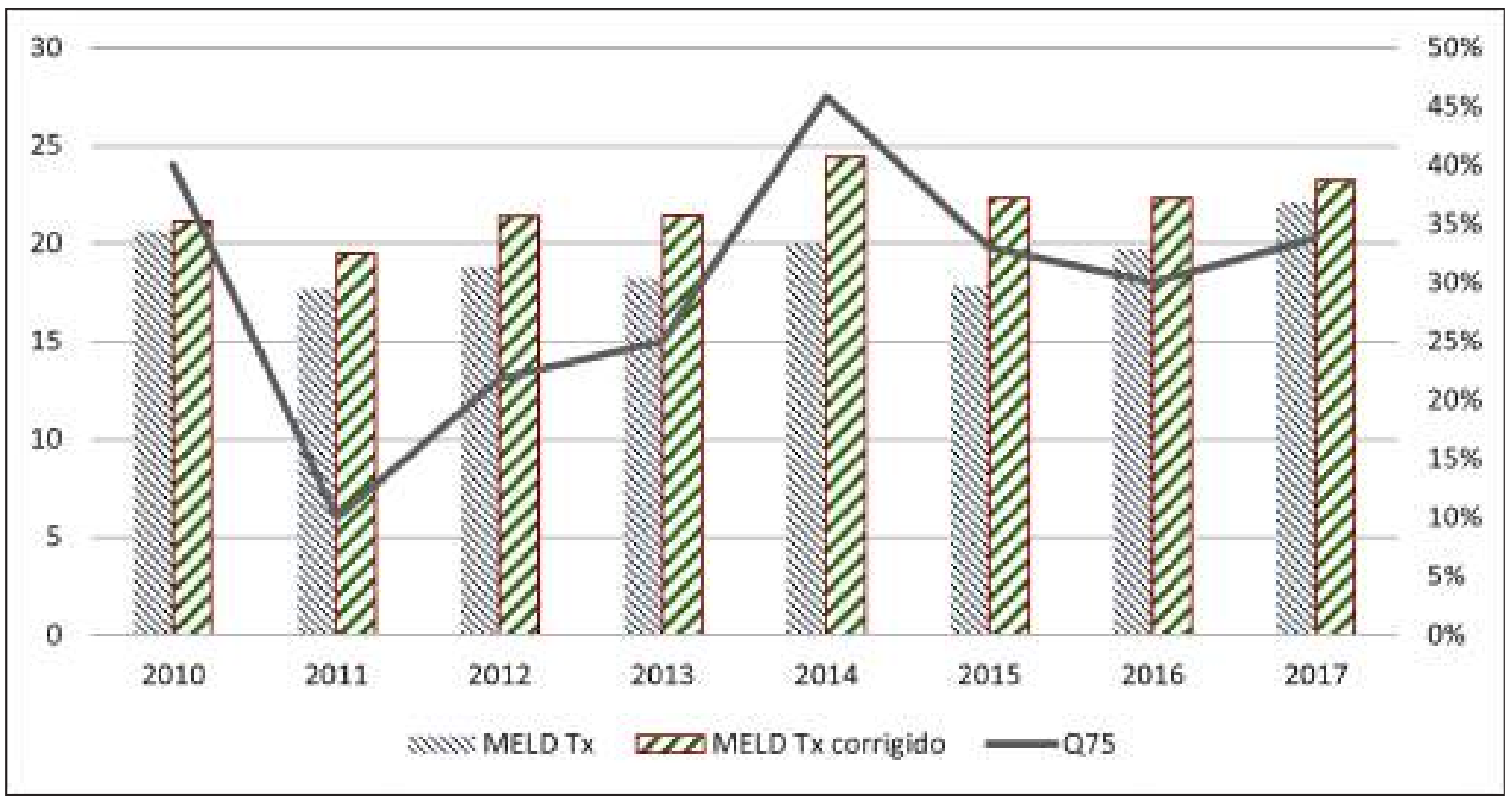


Tabela 4: Evolução anual de doadores efetivos e transplantes realizados.

\begin{tabular}{|c|c|c|c|c|c|c|c|c|c|c|}
\hline & 2010 & 2011 & 2012 & 2013 & 2014 & 2015 & 2016 & 2017 & 2018 & $\mathbf{p}$ \\
\hline $\begin{array}{l}\text { Doadores } \\
\text { (pmp) }\end{array}$ & $\begin{array}{c}93 \\
(8,9)\end{array}$ & $\begin{array}{c}112 \\
(10,7)\end{array}$ & $\begin{array}{c}151 \\
(14,5)\end{array}$ & $\begin{array}{c}191 \\
(18,3)\end{array}$ & $\begin{array}{c}173 \\
(16,6)\end{array}$ & $\begin{array}{c}241 \\
(21,7)\end{array}$ & $\begin{array}{c}345 \\
(30,9)\end{array}$ & $\begin{array}{l}427 \\
(38)\end{array}$ & $\begin{array}{c}540 \\
(47,7)\end{array}$ & - \\
\hline \multicolumn{11}{|c|}{ Total de transplantes } \\
\hline $\mathrm{N}$ & 11 & 48 & 91 & 100 & 91 & 126 & 208 & 265 & 304 & - \\
\hline Espera & $104 \pm 12$ & $279 \pm 44$ & $119 \pm 33$ & $112 \pm 13$ & $130 \pm 14$ & $155 \pm 20$ & $101 \pm 16$ & $69 \pm 11$ & $102 \pm 16$ & * \\
\hline Q75 & 247 & 283 & 100 & 143,5 & 159 & 177 & 120,5 & 82 & 119 & - \\
\hline \multicolumn{11}{|c|}{ Situação especial } \\
\hline $\begin{array}{l}N \\
(\% t x)\end{array}$ & 0 & $\begin{array}{c}4 \\
(8,33 \%)\end{array}$ & $\begin{array}{c}6 \\
(6,59 \%)\end{array}$ & $\begin{array}{c}13 \\
(13 \%)\end{array}$ & $\begin{array}{c}14 \\
(15,3 \%)\end{array}$ & $\begin{array}{c}17 \\
(13,4 \%)\end{array}$ & $\begin{array}{c}17 \\
(8,17 \%)\end{array}$ & $\begin{array}{c}7 \\
(2,6 \%)\end{array}$ & $\begin{array}{c}24 \\
(7,89 \%)\end{array}$ & - \\
\hline Espera & - & $139 \pm 64$ & $77 \pm 48$ & $101 \pm 47$ & $106 \pm 54$ & $181 \pm 182$ & $125 \pm 138$ & $69 \pm 70$ & $72 \pm 55$ & * \\
\hline Q75 & - & 177,5 & 133 & 139 & 149 & 197 & 166 & 147 & 111,5 & - \\
\hline \multicolumn{11}{|c|}{ Situação urgência } \\
\hline $\begin{array}{l}n \\
(\% \text { total } t x)\end{array}$ & $1(9,09 \%)$ & $2(4,16 \%)$ & $5(5,49 \%)$ & $5(5 \%)$ & $4(4,39 \%)$ & $2(1,58 \%)$ & $7(3,36 \%)$ & $13(4,9 \%)$ & $8(2,63 \%)$ & - \\
\hline Espera & 3 & $6 \pm 4$ & $2 \pm 2$ & $3 \pm 1$ & $11 \pm 10$ & $9 \pm 0,7$ & $1 \pm 0,9$ & $1,7 \pm 1,1$ & $2 \pm 1,30$ & * \\
\hline Q75 & 3 & 9 & 2 & 4 & 20 & 10 & 2 & 2 & 3 & - \\
\hline
\end{tabular}

${ }^{*} p<0,05$

Não houve diferença significativa entre o tempo médio de espera em lista para pacientes sem concessão $(112 \pm 197)$ e com concessão $(110 \pm 107)$ de situação especial $(p>0,05)$.
O padrão de dimensão temporal do tempo de espera em lista no percentil 75 (Q75) em relação ao número de transplantes está demonstrado na figura 3.

Figura 3: Padrão temporal do tempo de espera em lista de pacientes com e sem situação especial.

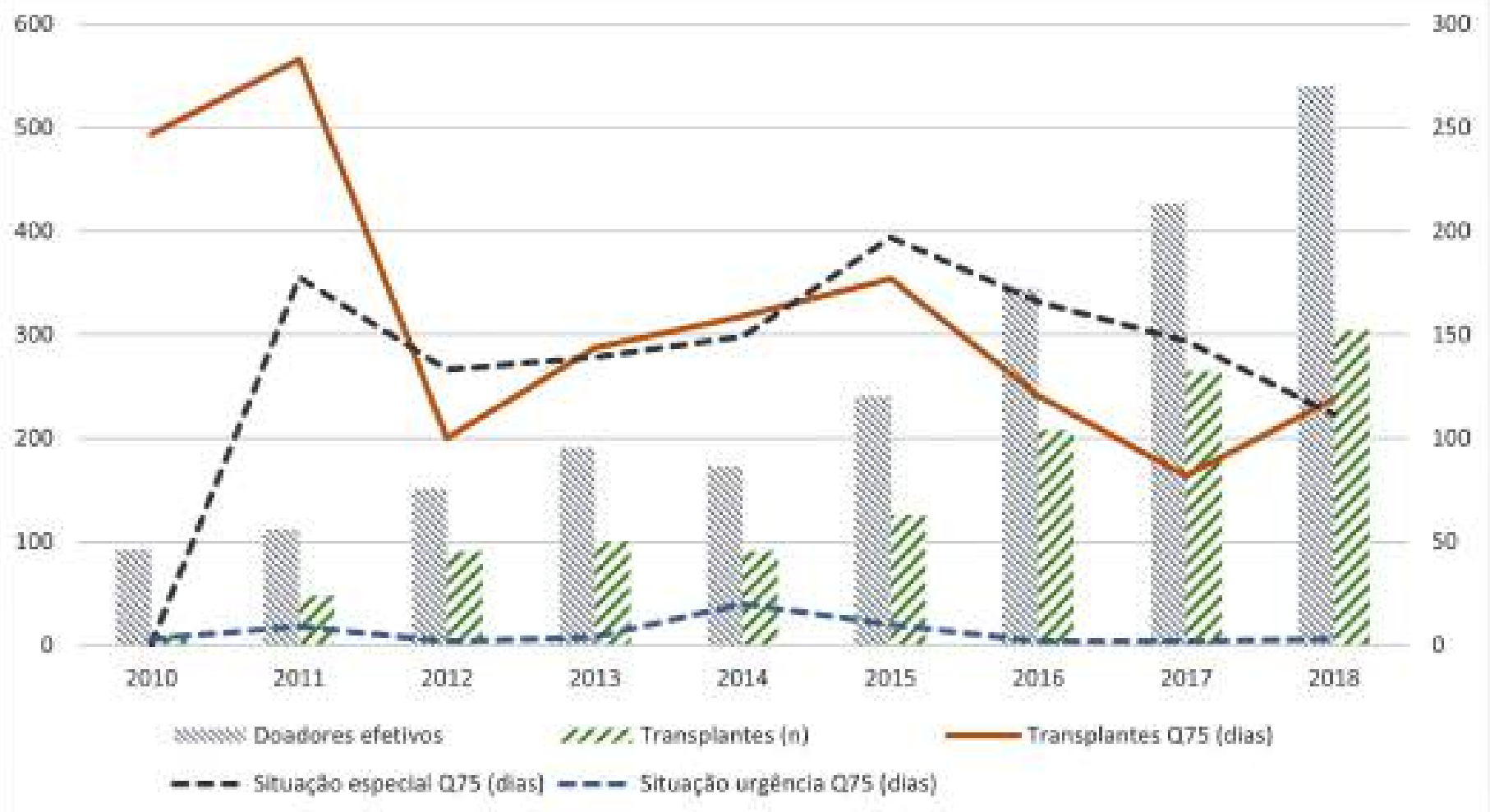




\section{DISCUSSÃO}

A alocação de órgãos para transplante envolve princípios éticos de equidade - tratamento igual para necessidades iguais, priorização para casos graves - e utilidade, objetivando salvar o maior número de vidas possível. ${ }^{9}$ Esse equilíbrio utilidade-equidade é difícil de ser obtido. O atual sistema de alocação baseado no MELD atinge o objetivo de ser de fácil compreensão e transparente, tendo modificado substancialmente os transplantes de fígado de maneira substancial, ${ }^{1}$ nos mais variados países em que foi adotado. ${ }^{10}$

O sistema inclui a previsão de concessão de pontos de bonificação para situações especiais, medida essa tomada para ajustar o potencial de mortalidade em lista de espera para diferentes etiologias de doenças hepática passíveis de transplante. ${ }^{11}$

Observamos em nosso estudo um predomínio da etiologia de doença hepática alcoólica, seguida das infecções pelos vírus da hepatite $B$ e $C$, proporção observada em outros estudos de população brasileira, ${ }^{4}$ porém diferente da população norte-americana, onde predomina a infecção pelo vírus da hepatite C. ${ }^{12}$ Essa proporção não se modifica nos extratos de doença mais grave representada pelo MELD, i.e. acima do Q75.

A maturidade do sistema MELD traz consigo um aumento da proporção de pacientes transplantados com doença mais grave, ${ }^{1}$ tendência observada em nossa população (figura 2). Esse aumento do MELD, necessário para transplante apontado pela literatura, tem como fator influenciador, apesar de não ser o único, o sistema de concessão de pontos de exceção para as situações especiais. ${ }^{13} \mathrm{O}$ ingresso de pacientes com situação especial e seu progressivo aumento do MELD com o passar do tempo em lista resulta em maiores taxas de transplante e menor tempo de espera para esse subgrupo de pacientes. ${ }^{6,11}$

Entretanto, menor tempo de lista de espera para o grupo de situação especial não foi observada em nossa população (figura 3). A explicação para essa dissonância com as evidências da literatura internacional e até com os achados de outros estados brasileiros, reside no fato da contínua expansão da oferta de órgãos. Nesse cenário de crescimento de oferta, mesmo o aumento da demanda (maior número de transplantes) acabou não impactando no tempo de espera em lista. Constatase um tempo de espera menor do que seis meses em $83,78 \%$ dos casos no PR, frente a $60 \%$ dos casos nos EUA. ${ }^{12}$

Ao adicionarmos a observação que, proporcionalmente, o estado do PR tem baixo índice de transplante por situações especiais $(8,17 \%)$, com frequência acima de $30 \%$ em outros estados brasileiros ${ }^{1,14,15}$ e nos EUA, ${ }^{6,12}$ a "pressão" em lista pelas situações especiais ainda não se tornou um problema em nossa realidade.

\section{CONCLUSÃO}

Concluímos que o impacto do progressivo aumento do MELD e tempo de espera em lista secundário à concessão de situações especiais, não é observado na lista de espera do estado do Paraná. Essa discrepância, provavelmente, é secundária ao contínuo crescimento de maneira quase paralela do número de doadores e transplantes realizados e o baixo número de transplantes sob concessão de situação especial.

\section{ABSTRACT}

MELD system is settled in Brazil. The increasing MELD to the allocation, the influence of different etiologies, and the imbalance on the list, secondary to the granting upon special situations have raised discussions towards modernization of the system. In Brazil, each state is responsible by organizing the organ harvesting, resulting in a heterogeneous system. Purpose. To analyze the waiting time and MELD required for transplantation, the impact of different etiologies and granting upon special situations in the state of Paraná. Method. Analysis of 1248 adult liver transplants between 2010 and 2018, stratified according to the etiology, MELD and waiting time. Results. Age of $52 \pm 11.4$ years. $112 \pm 197$ days, $83.78 \%$ waiting time on the list of transplant cases below 6 months. Special situations were granted in $8.25 \%$ $(n=103)$ of cases. There was no significant difference as to the waiting time on the list of the group with (110 \pm 107$)$ and without (112 \pm 197$)$ special situation. Transplant MELD (19.69 \pm 7.86$)$, corrected MELD (22.24 \pm 6.42$)$. Although being different among groups of etiology, MELD did not present growth pattern over time. Alcohol etiology $(27.08 \%)$ and viral infections (23.56\%) were the most frequent. $29.75 \%$ of transplants were performed with MELD above $75 \%$. There was no change in the most common etiologies in this subgroup. Organ supply increased from 8.9pmp (2010) to 47.7pmp (2017). Conclusion. The impact of the progressive increase of MELD and waiting time on a list, secondary to the granting upon special situations is not observed on the waiting list of the State of Paraná. This discrepancy is probably secondary to the continuous growth almost parallel to the amount of donors and transplants performed.

Keywords: Liver Transplantation; Waiting Lists; Technology Applied to Waiting Lists; Risk Index. 


\section{REFERÊNCIAS}

1. Machado AGS, Fleck AM, Jr, Marroni C, Zanotelli ML, Cantisani G, de Mello Brandao AB. Impact of MELD score implementation on liver allocation: experience at a Brazilian center. Ann Hepatol. 2013 May-Jun;12(3):440-7.

2. Freeman RB, Jr. The model for end-stage liver disease comes of age. Clin Liver Dis. 2007 May;11(2):249-63.

3. Silveira F, Silveira FP, Macri MM, Nicoluzzi JE. [Analysis of liver waiting list mortality in Parana, Brazi: what shall we do to face organ shortage?]. Arq Bras Cir Dig. 2012 Apr-Jun;25(2):110-3.

4. Freitas AC, Itikawa WM, Kurogi AS, Stadnik LG, Parolin $\mathrm{MB}$, Coelho JC. The impact of the model for endstage liver disease (MELD) on liver transplantation in one center in Brazil. Arq Gastroenterol. 2010 JulSep;47(3):233-7.

5. Bahra M, Neuhaus P. Liver transplantation in the high MELD era: a fair chance for everyone? Langenbecks Arch Surg. 2011 Apr;396(4):461-5.

6. Northup PG, Intagliata NM, Shah NL, Pelletier SJ, Berg $\mathrm{CL}$, Argo CK. Excess mortality on the liver transplant waiting list: unintended policy consequences and Model for End-Stage Liver Disease (MELD) inflation. Hepatology. 2015 Jan;61(1):285-91.

7. RBT - Registro Brasileiro de Transplantes. ABTO Associação Brasileira de Transplante de Órgãos, 2018.

8. Dean AG AT, Sunki GG, Friedman R, Lantinga M, Sangam S, Zubieta JC, et al. Epi Info ${ }^{\mathrm{TM}}$, a database and statistics program for public health professionals. CDC, Atlanta, GA, USA. 2011.
9. Vitale A, Volk M, Cillo U. Urgency, utility, and time horizon of transplant benefit. Liver Transpl. 2015 Apr;21(4):565-6.

10. Toso C, Mazzaferro V, Bruix J, Freeman R, Mentha G, Majno P. Toward a better liver graft allocation that accounts for candidates with and without hepatocellular carcinoma. Am J Transplant. 2014 Oct;14(10):2221-7.

11. Pillai A, Couri T, Charlton M. Liver Allocation Policies in the USA: Past, Present, and the Future. Dig Dis Sci. 2019 Apr;64(4):985-92.

12. Kim WR, Lake JR, Smith JM, Schladt DP, Skeans MA, Noreen SM, et al. OPTN/SRTR 2017 Annual Data Report: Liver. Am J Transplant. 2019 Feb;19 Suppl 2:184-283.

13. Roayaie K, Feng S. Allocation policy for hepatocellular carcinoma in the MELD era: room for improvement? Liver Transpl. 2007 Nov;13(11 Suppl 2):S36-43.

14. Monteiro F, Coria SA, Boni R, Pereira LA. Model for end-stage liver disease: impact of the new deceased donor liver allocation policy in Sao Paulo, Brazil. Transplant Proc. 2009 JanFeb;41(1):226-8.

15. Martino RB, Waisberg DR, Dias APM, Inoue VBS, Arantes RM, Haddad LBP, et al. Access to Liver Transplantation in Different ABO-Blood Groups and "Exceptions Points" in a Model for End-Stage Liver Disease Allocation System: A Brazilian Single-Center Study. Transplant Proc. 2018 Apr;50(3):754-7.

\section{AGRADECIMENTOS}

Os autores gostariam de agradecer às famílias dos doadores que expressaram autorização para doação dos órgãos. Ao Sistema Estadual de Transplantes e a todos os coordenadores das OPOs e CIHDOTTs do estado do Paraná que através do esforço

incansável permitem a identificação e realização de todo o processo que permite a existência de um doador efetivo. 